\title{
Tl.163.1
}

\section{Linking SSO Systems Working Group Charter}

- PDF: LinkingSSOSystemsWorkingGroupCharter.pdf

- Text: LinkingSSOSystemsWorkingGroupCharter.txt

\section{More Information}

\begin{tabular}{|l|l|}
\hline Repository ID & TI.163.1 \\
\hline Persistent URL & http://doi.org/10.26869/TI.163.1 \\
\hline Title & Linking SSO Systems Working Group Charter \\
\hline Authors & Rob Carter, https://orcid.org/0000-0002-5903-5799 \\
Keith Wessel, https://orcid.org/0000-0002-8047-3187 \\
\hline Sponsor & CACTI \\
\hline Review & \\
\hline Status & Preserve \\
\hline Publish Date & February, 2022 \\
\hline DOI & $10.26869 / T I .163 .1$ \\
\hline Signature & \\
\hline Deprecated & No \\
\hline Future Review & \\
\hline Supersedes & \\
\hline Format & PDF, Text \\
\hline Related Docs & \\
\hline Development Location & \\
\hline IP Framework & CC BY 4.0 \\
\hline Subject Tags & cacti, charter \\
\hline Notes & \\
\hline
\end{tabular}

